\title{
Specification and Implementation of Dynamic Web Site Benchmarks
}

\author{
Cristiana Amza, Anupam Chanda, Alan L. Cox, \\ Sameh Elnikety, Romer Gil, Karthick Rajamani* \\ and Willy Zwaenepoel \\ CS Department - Rice University \\ *IBM Austin Research Lab \\ \{amza, anupamc, alc, sameh, rgil,willy\}@rice.edu, \\ karthick@us.ibm.com
}

\begin{abstract}
The absence of benchmarks for Web sites with dynamic content has been a major impediment to research in this area. We describe three benchmarks for evaluating the performance of Web sites with dynamic content. The benchmarks model three common types of dynamic content Web sites with widely varying application characteristics: an online bookstore, an auction site, and a bulletin board. For the online bookstore, we use the TPC$\mathrm{W}$ specification. For the auction site and the bulletin board, we provide our own specification, modeled after ebay.com and slahdot.org, respectively. For each benchmark we describe the design of the database and the interactions provided by the Web server.

We have implemented these three benchmarks with a variety of methods for building dynamic-content applications, including PHP, Java servlets and EJB (Enterprise Java Beans). In all cases, we use commonly used open-source software. We also provide a client emulator that allows a dynamic content Web server to be driven with various workloads. Our implementations are available freely from our Web site for other researchers to use.

These benchmarks can be used for research in dynamic Web and application server design. In this paper, we provide one example of such possible use, namely discovering the bottlenecks for applications in a particular server configuration. Other possible uses include studies of clustering and caching for dynamic content, comparison of different application implementation methods, and studying the effect of different workload characteristics on the performance of servers. With these benchmarks we hope to provide a common reference point for studies in these areas.
\end{abstract}

\section{Introduction}

Web content is increasingly generated dynamically, a departure from the early days of the Web when virtually all content consisted of static HTML or image files. Dynamic Web content is typically generated by a combination of a front-end Web server, an application

\author{
Emmanuel Cecchet and Julie Marguerite
}

CS Department - Rice University/
INRIARhone-Alpes
emmanuel.cecchet@inrialpes.fr
julie.marguerite@inrialpes.fr

server and a back-end database (see figure 1). The (dynamic) content of the site is stored in the database. The application server provides methods that implement the business logic of the application. As part of that, the application typically accesses the database. The three servers (Web, application and database server) may all execute on a single machine, or each one of them may execute on a separate machine or on a cluster of machines, or various combinations thereof.

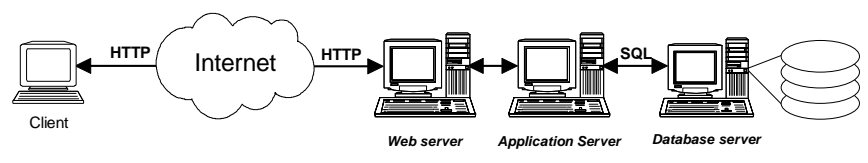

Figure 1. Typical Configuration of a Dynamic Content Web Site

To study the architecture and the performance of dynamic Web sites, benchmarks are needed that are representative of some of the common applications for such Web sites, yet simple enough to be understood and manipulated with ease. TPC-W [24] provides a specification for benchmarking e-commerce applications. It models an online bookstore, such as amazon.com. This paper proposes new specifications for two different types of dynamic content sites: auction sites and bulletin boards. Our benchmark for auction sites is modeled after eBay [7]. For bulletin boards, our benchmark is modeled after the Slashcode [20], which is used in many bulletin board sites including its originator site Slashdot [21].

We have implemented all three applications using a variety of methods. In particular, we have implementations using the PHP Web-scripting language [18], Java servlets [9], and different ways of using EJB (Enterprise Java Beans) [8]. The implementations use open-source software platforms such as the Apache Web server, the Tomcat servlet server, the JOnAS and JBoss EJB servers, and the MySQL relational database $[1,2,10$, $12,15]$. The source code for all of these implementations is available on our web site http://www.cs.rice.edu/CS/Systems/DynaServer. The choice of an open-source platform allows easy use of our benchmarks by other researchers. In addition to the server 
applications, we have also developed a client emulator to drive a dynamic content Web server with various workloads.

These benchmarks can be used for a variety of different studies on dynamic Web content generation. We have already used the auction site to compare various application implementation methods, container designs and communication optimizations for EJB applications [6]. We have also used all three applications in a comparison of PHP, Java servlets and EJB [5]. Currently, we are using the benchmarks in studies on clustering and caching for dynamic content.

As one example of the type of work that can be performed with these benchmarks, we present a bottleneck analysis of the PHP versions of the applications using PC server hardware. In all our experiments, the Web server and the database run on a separate machine. In particular, we use a $1.33 \mathrm{GHz}$ AMD Athlon with $768 \mathrm{MB}$ memory and a 60GB disk for each machine. The two machines are connected to each other and to a set of machines running client emulation software by a switched 100Mbps Ethernet. For the online bookstore the CPU on the database server is the bottleneck. In contrast, for the auction site and the bulletin board the Web server CPU is the bottleneck. In none of the experiments we found the memory, the disk or the network to be a bottleneck. We also comment on the effect of enforcing various degrees of (transactional) consistency in the benchmarks.

The rest of this paper is structured as follows. Sections 2 to 4 describe the benchmark specifications. Section 5 describes the client emulator tool. Section 6 outlines the example use of our benchmarks discussed in this paper. Section 7 describes our experimental environment, both in terms of software, hardware, workloads and application sizing. Sections 8 to 10 analyze the results for the three benchmarks. We cover related work in section 11, and conclude in section 12 .

\section{Online Bookstore Benchmark}

The TPC-W benchmark from the Transaction Processing Council [24] is a transactional Web benchmark specifically designed for evaluating e-commerce systems. Our online bookstore benchmark is modeled after TPCW. It implements all the functionality specified in TPC-W that has an impact on performance, including transactional consistency and support for secure transactions. It does not implement some functionality specified in TPC-W that has an impact only on price and not on performance, such as the requirement to provide enough storage for 180 days of operation.

All persistent data, with the exception of the images used with each book, is stored in the database. The database contains eight tables: customers, addresses, orders, order_line, credit_info, items, authors, and countries. The order_line, orders and credit_info tables store information about orders that have been placed, in particular: the book ordered, the quantity and discount (table order_line), the customer identifier, date of order, information about the amount paid, shipping address and status (table orders), and credit card information such as type, number and expiration date (table credit_info). The items and authors tables contain information about the books and their authors. Customer information, including real name and user name, contact information (email, address), and password, is obtained via a customer registration form and maintained in the customers and addresses tables.

Each item in the database has two corresponding images: a full size image and a thumbnail image. The images are stored on the Web server. We implemented the 14 different interactions specified in the TPC-W benchmark specification. Of the 14 scripts, 6 are readonly, while 8 cause the database to be updated. The readonly interactions include access to the home page, listing of new products and best sellers, requests for product detail, and two interactions involving searches. Read-write interactions include user registration, updates to the shopping cart, two interactions involving purchases, two involving order inquiry and display, and two involving administrative tasks. We use the same distribution of script execution as specified in TPC-W. An interaction may also involve requests for multiple embedded images, each image corresponding to an item in the inventory. With one exception, all interactions query the database server.

We implement a Payment Gateway Emulator (PGE), which represents an external system that authorizes payment of funds during purchasing interactions [24, clause 6.4]. The Web server contacts the PGE using an SSL session to send the credit card information. The PGE replies with a message containing the authorization number. The PGE is not a part of the benchmarked system.

\section{Auction Site Benchmark}

Our auction site benchmark implements the core functionality of an auction site: selling, browsing and bidding. We do not implement complementary services like instant messaging or newsgroups. We distinguish between three kinds of user sessions: visitor, buyer and seller. For a visitor session, users need not register but are only allowed to browse. Buyer and seller sessions require registration. In addition to the functionality provided during visitor sessions, during a buyer session users can bid on items and consult a summary of their current bids, their rating and comments left by other users. Seller sessions require a fee before a user is allowed to put up an item for sale. An auction starts immediately and lasts 
typically for no more than a week. The seller can specify a reserve (minimum) price for an item.

The database contains seven tables: users, items, bids, buy_now, comments, categories and regions. The users table records contain the user's name, nickname, password, region, rating and balance. Besides the category and the seller's nickname, the items table contains the name that briefly describes the item and a more extensive description, usually an HTML file. Every bid is stored in the bids table, which includes the seller, the bid, and a max_bid value used by the proxy bidder (a tool that bids automatically on behalf of a user). Items that are directly bought without any auction are stored in the buy_now table. The comments table records comments from one user about another. As an optimization, the number of bids and the amount of the current maximum bid are stored with each item to prevent many expensive lookups of the bids table. This redundant information is necessary to keep an acceptable response time for browsing requests. As users only browse and bid on items that are currently for sale, we split the item table in a new and an old item table. The very vast majority of the requests access the new items table, thus considerably reducing the database's working set.

Our auction site defines 26 interactions. Among the most important ones are browsing items by category or region, bidding, buying or selling items, leaving comments on other users and consulting one's own user page (known as myEbay on eBay [7]). Browsing items also includes consulting the bid history and the seller's information.

\section{Bulletin Board Benchmark}

Our bulletin board benchmark is modeled after an online news forum like Slashdot [21]. We originally considered using the Perl-based Slashcode [20], which is freely available, but we concluded that the code was too complex to serve as a benchmark. Instead, we implement the essential bulletin board features of the Slashdot site. In particular, as in Slashcode, we support discussion threads. A discussion thread is a logical tree, containing a story at its root and a number of comments for that story, which may be nested. Users have two different levels of authorized access: regular user and moderator. Regular users browse and submit stories and comments. Moderators, in addition, review stories and rate comments.

The main tables in the database are the users, stories, comments, and submissions tables. The users table contains each user's real name and nickname, contact information (email), password, level of authorized access, and rating. The stories table contains each story's title and body, the nickname of the story's author, the date the story was posted, the number of comments at the outermost nesting level, and the category the story fits under. The categories table contains the same categories as the Slashdot site. The comments table contains the comment's subject and body, the nickname of the comment's author, the date the comment was posted, the identifier of the story or the parent comment it belongs to, and a comment rating. Each submitted story is initially placed in the submissions table, unless submitted by a moderator. We maintain a moderator_log table, which stores the moderator ratings for comments. Regular user ratings are computed based on the ratings for the comments they have posted.

For efficiency reasons, we split both the stories and comments tables into separate new and old tables. In the new stories table we keep the most recent stories with a cut-off of one month. We keep old stories for a period of two years. The new and old comments tables correspond to the new and old stories respectively. The majority of the browsing requests are expected to access the new stories and comments tables, which are much smaller and therefore much more efficiently accessible. A daemon is activated periodically to move stories and comments from the new to the old tables as appropriate.

We have defined 24 Web interactions. The main ones are: generate the stories of the day, browse new stories, older stories, or stories by category, show a particular story with different options on filtering comments, search for keywords in story titles, comments and user names, submit a story, add a comment, review submitted stories and rate comments at the moderator level. Full text search is currently not supported. Without additional support, it requires a prohibitive processing time in a generalpurpose relational database. Typically, an external search engine would be used to perform this task.

\section{The Client Emulator: a Workload Generation Tool}

In addition to the benchmarks described in Sections 2 to 4, we have also developed a client emulator that implements a methodology for generating workloads for dynamic content sites. The tool is reasonably general and can be extended to other benchmarks of the same nature. The prototype tool that we have implemented requires some code to be written specific to the application under test, although much of that could be automated.

Following the TPC-W specifications, the workload generated by the client emulator consists of a number of concurrent clients and their interactions with the system under test (SUT). The number of clients emulated by the tool can be varied to vary the load on the SUT.

Each emulated client opens a session with the SUT. The session remains alive for a period of time, called session time, at the end of which the connection is closed. Each session is a persistent HTTP connection with the 
SUT. Using this connection, the client repeatedly makes a request, parses the server's response to the request, and follows a (hyper-)link embedded in the response. The server's response is a Web page consisting of an answer to the query in the last request and the links to the set of pages that the client can transition to from this response. For example, after viewing the home page of TPC-W, the client may decide to view the best-selling books of some particular subject, and hence the response to the home page request contains a link for the best-sellers, in addition to other links. A Markov model determines which subsequent link from the response to follow. The Markov model uses a transition probability matrix with probabilities attached to transitions from one state to another. A state in the transition matrix corresponds to a particular interaction of the SUT and its web page, e.g., home page, best-sellers page, product information page, etc. A transition corresponds to clicking on a link in the page. The client emulator waits for an amount of time, called the think time, before initiating the next interaction. This emulates the "thinking" period of a real client who takes a period of time before clicking on the next request.

The tool takes as its input a number of clients and a transition matrix. A particular column of the transaction matrix specifies the probabilities to transition from a given page to all other pages/states. The tool provides certain consistency checks on the values in the table, guaranteeing that probabilities in any column add up to 1 .

The tool collects system utilization statistics (CPU, memory, network bandwidth, etc.) on the machines of the SUT specified in a configuration file. At the end of the execution, the tool displays detailed statistics about the execution, including, in particular, overall throughput and response time statistics, and CPU, memory, network and disk utilization graphs for the length of the run (as shown in later sections).

\section{Example Use of the Benchmarks}

In the rest of this paper, we demonstrate the use of these benchmarks by performing a bottleneck analysis of the applications. We vary the load on the system and monitor the resulting throughput. We focus on measuring the throughput against system load and the utilization of various resources, rather than on other performance metrics such as response time.

We use the PHP version of the benchmarks, with the Web server (including the PHP module) executing on one machine and the database executing on another machine. We use Apache as the Web server and MySQL as the database server. This setup is quite common for smaller web sites. The most recent Netcraft survey [16] showed that $60 \%$ of all Web sites are running Apache. About $40 \%$ of these sites had the PHP module compiled in.
Bottleneck analysis allows the site maintainer to predict which parts of the overall system need to be upgraded to deal with increased load, for instance, by using a faster machine, an SMP machine or a cluster.

\section{Hardware and Software Environment}

\subsection{Software Environment}

We use Apache v.1.3.22 as the Web server, configured with the PHP v.4.0.6 module, mod_ssl version 2.8.5 and openSSL 0.9.5a. We increase the maximum number of Apache processes to 512. We observe that with that value, the number of Apache processes is never a limit on performance. We use MySQL v.3.23.43-max as our database server. We use MyISAM and BDB tables as non-transactional and transactional database tables, respectively. All machines run the 2.4.12 Linux kernel.

In MySQL, as soon as overload occurs, performance degrades. In particular, performance degrades faster when the number of update queries in the workload becomes larger. Transactions are a new and relatively less stable addition to MySQL. Transactions exacerbate the performance degradation under heavy load. The effects of these limitations in MySQL will be seen in several experiments reported in sections 8 to 10 .

\subsection{Transactional Semantics}

We study two levels of transactional consistency.

The first level provides transactional isolation, but does not provide for transactional durability and atomicity. For all interactions (PHP scripts) that contain update queries, we insert database lock operations that obtain all locks necessary for all queries in a script (for both read and write operations) before the first query. Locks are held until the end of the script's execution. Scripts that contain only read-only queries do not obtain locks. Unless mentioned otherwise, this level of consistency is the default used in our experiments.

The second level provides full ACID transaction guarantees. For all interactions (PHP scripts) we insert a begin_transaction before the first database query is issued and an end_transaction after the last query is issued. If a script fails, an abort can be issued to release any database locks that are held and to undo all modifications to the database. We refer to this second level of consistency as "with transactions" in the experiments in sections 8 to 10.

\subsection{Hardware Platform}

The Web server and the database server run on an AMD Athlon $1.33 \mathrm{GHz}$ CPU with 768MB SDRAM, and a Maxtor 60GB 5,400rpm disk drive. A number of $800 \mathrm{MHz}$ AMD Athlon machines run the client emulation software. We use enough client emulation machines to make sure that the clients do not become a bottleneck in any of our 
experiments. All machines are connected through a switched 100Mbps Ethernet LAN.

\subsection{Measurement Methodology}

Each experiment is composed of 3 phases. A warm-up phase initializes the system until it reaches a steady-state throughput level. We then switch to the steady-state phase during which clients machines perform all measurements. Finally, a cool-down phase allows for small time differences experienced by different client machines to be smoothed out and slows down the incoming request flow until the end of the experiment. For all experiments with a particular application we use the same length of time for each phase, but the duration of each phase is different for different applications. The online bookstore uses 1 minute, 10 minutes and 30 seconds for the warm-up, the steady-state and the cool-down phase, respectively. The auction site uses 5, 30 and 5 minutes, and the bulletin board 2.5, 15 and 2.5 minutes. These values are chosen based on observation of the length of time before the experiment reaches a steady state, and the length of time necessary to obtain reproducible results.

To measure the load on each machine, the client emulator invokes the sysstat utility [23] that every second collects CPU, memory, network and disk usage from the Linux kernel.

\subsection{Workloads and Application Sizing}

TPC-W specifies three different workload mixes, differing in the ratio of read-only to read-write scripts. The browsing mix contains $95 \%$ read-only scripts, the shopping $\operatorname{mix} 80 \%$, and the ordering mix $50 \%$. The database contains 10,000 items and the corresponding 183 $\mathrm{MB}$ of data for the item images are stored on the Web server. We use two database sizes by controlling the number of customers: a large database (3.5GB) and a small database (350MB) that fits entirely in memory on our experimental platform. These sizes include the necessary database indices.

For the auction site, we use two workload mixes: a browsing mix made up of only read-only interactions and a bidding mix that includes $15 \%$ read-write interactions. We size our system according to some observations found on the eBay Web site. We always have about 33,000 items for sale, distributed among eBay's 40 categories and 62 regions. We keep a history of 500,000 auctions in the old-items table. There is an average of 10 bids per item, or 330,000 entries in the bids table. The buy_now table is small, because less than $10 \%$ of the items are sold without auction. The users table has 1 million entries. We assume that users give feedback (comments) for $95 \%$ of the buying transactions. The new and old comments tables therefore contain about 31,500 and 475,000 comments, respectively. The total size of the database, including indices, is $1.4 \mathrm{~GB}$.

For the bulletin board, we use two workload mixes: a browsing mix and a submission mix. The browsing mix is a read-only workload that does not allow users to post stories or comments. The submission mix contains $85 \%$ read-only interactions, with the remaining $15 \%$ being story and comment submissions and moderation interactions. The two mixes correspond to what we observed as the two extremes in workload characteristics for real bulletin board sites. The browsing mix corresponds to Slashdot's workload, a site with a large user base in which $99.5 \%$ of accesses are reads [22]. The submission load corresponds to that of a bulletin board with a relatively small user base that posts more frequently [25]. On this bulletin board about 10 to $15 \%$ of all accesses are writes. We generate the story and comment bodies with words from a given dictionary and lengths between $1 \mathrm{~KB}$ and $8 \mathrm{~KB}$. Short stories and comments are much more common, so we use a Zipf-like distribution for story length [3]. The database contains 2 years of stories and comments. We use an average of 15 to 25 stories per day and between 20 and 50 comments per story, as we observed on Slashdot. We emulate 500,000 total users, out of which $10 \%$ have moderator access privilege. With these parameters, the database size is 439MB. We also created a larger database of $1.4 \mathrm{~GB}$ containing more old stories and comments. The results are very similar as the majority of the requests access the new stories and comments.

For all benchmarks, we use the think time and the session time specified by TPC-W, 7 seconds and 15 minutes, respectively

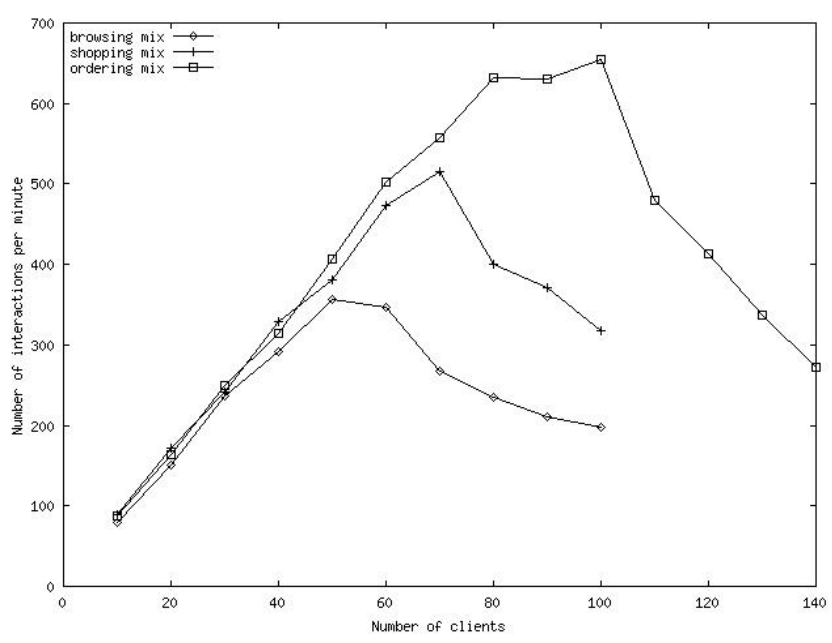

Figure 2. Online bookstore throughput in interactions per minute as a function of number of clients. 


\section{Bottleneck Analysis for Online Bookstore Benchmark}

Figure 2 shows the throughput, in interactions per minute, as the number of clients increases, for each of the three workload mixes and for the small database. The peak throughputs are 356,515 , and 654 interactions per minute, for the browsing, shopping, and ordering mix, respectively. Figure 3 to figure 5 show, for the different mixes, the average CPU utilization on the Web server and the database server as the number of clients increases.

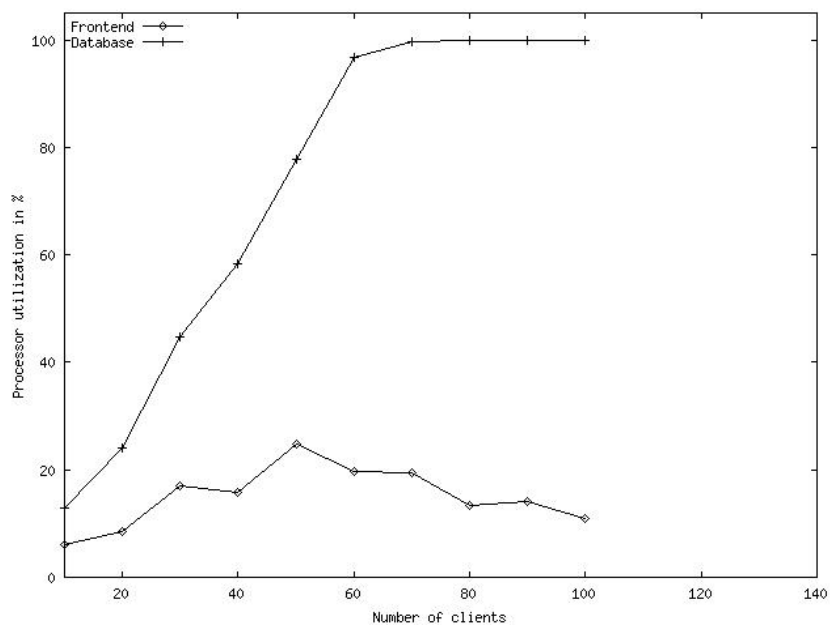

Figure 3. Online bookstore percentage CPU utilization as a function of number of clients for the browsing mix.

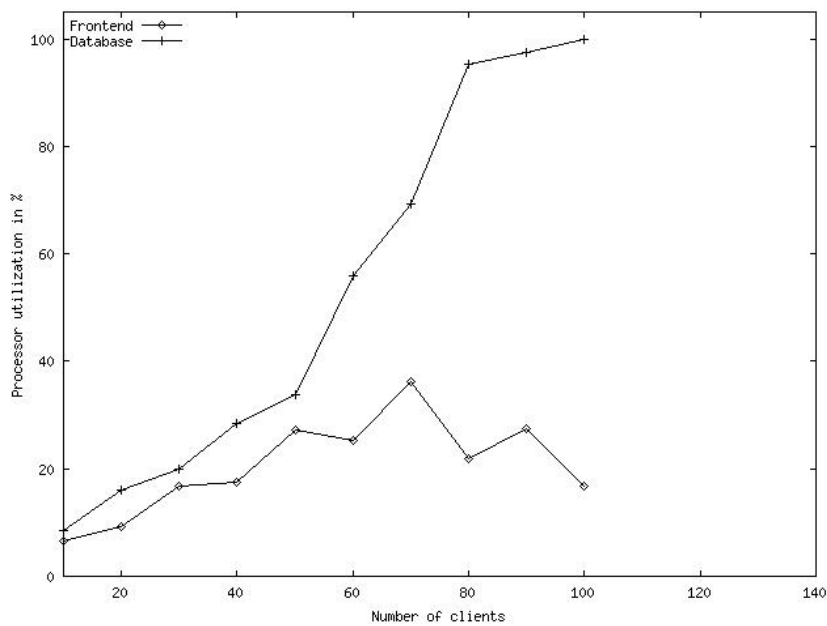

Figure 4. Online bookstore percentage CPU utilization as a function of number of clients for the shopping mix.

From these figures we conclude that for all workload mixes, the CPU on the database machine is the bottleneck resource at the peak throughput. The complex nature of many of the database queries makes the database the bottleneck. In comparison, the cost of handling and executing the PHP scripts for these interactions on the
Web server is small. The read-only queries are, on average, more complex than the read-write queries. Hence, for workload mixes with a larger number of readonly queries, overall throughput is lower and the database is more of a bottleneck.

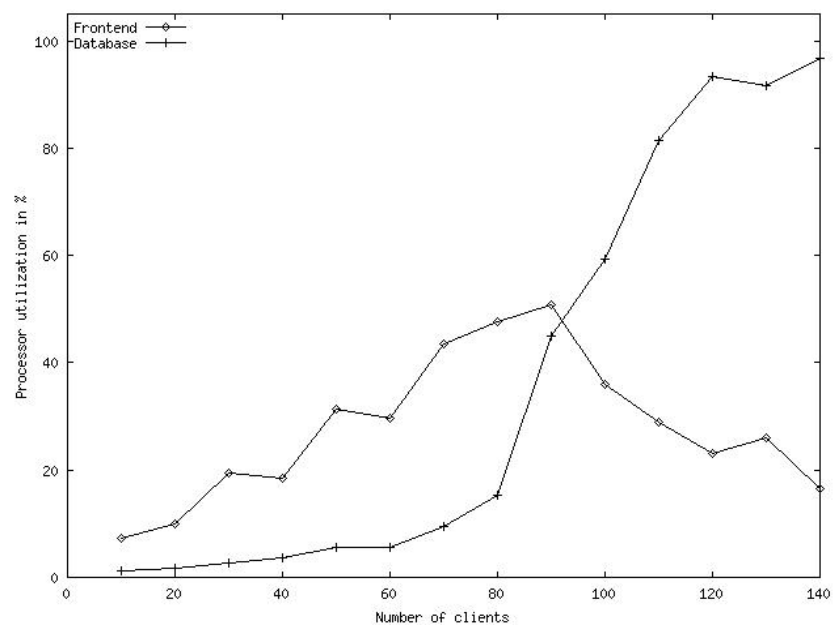

Figure 5. Online bookstore percentage CPU utilization as a function of number of clients for the ordering mix.

We monitor the memory usage and disk access on the Web server and the database throughout all our experiments. None of these resources is the bottleneck.

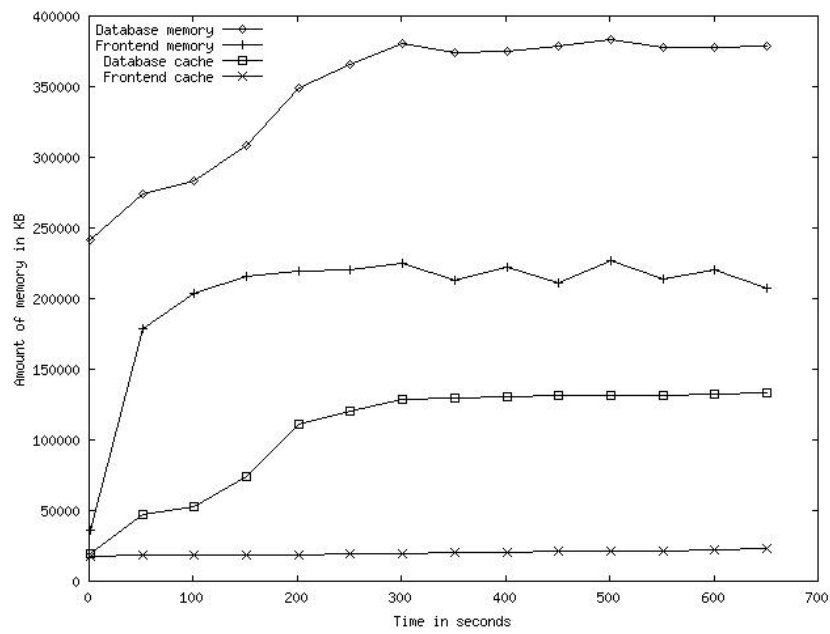

Figure 6. Online bookstore memory usage in $\mathrm{KB}$ as a function of time at the peak throughput for the ordering mix.

Figure 6 and figure 7 show the utilization of memory and disk for the ordering mix at its peak throughput, which is also the highest throughput for any of the three mixes. During a short initial transient period, the database reads information from the disk to warm up its cache. After this period, the working set fits in memory and hence disk access is low. Memory utilization in steady 
state is approximately $200 \mathrm{MB}$ on the Web server and $390 \mathrm{MB}$ on the database.

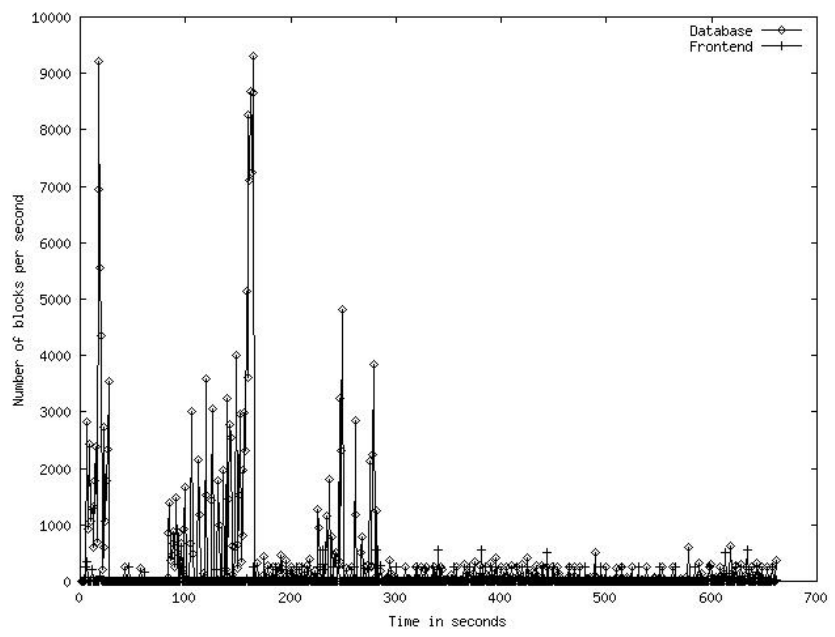

Figure 7. Online bookstore disk usage in number of blocks per seconds as a function of time at the peak throughput for the ordering mix.

Figure 8 shows the network usage between the database and the Web server, and between the Web server and the clients. The latter is on average $3.2 \mathrm{Mb} / \mathrm{s}$, while the former is always lower than $1.6 \mathrm{Mb} / \mathrm{s}$. Clearly, neither of these forms a bottleneck.

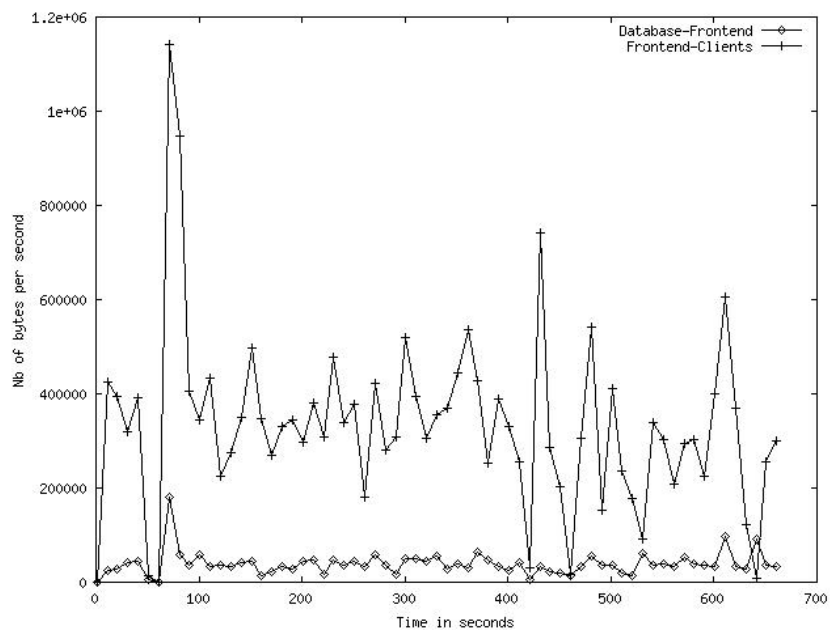

Figure 8. Online bookstore network usage in bytes/s as a function of time at the peak throughput for the ordering mix.

As expected, when we add full transaction semantics, the throughput for all mixes is lower. In particular, the peak throughputs are 240,395 and 191 interactions per minute for the browsing, shopping and ordering mix, respectively. The database CPU remains the bottleneck. When we use the larger database, the disk utilization on the database server becomes higher, but the database CPU remains the bottleneck. We obtain peak throughputs of 56, 120 , and 494 interactions per minute, for the browsing, shopping, and ordering mix, respectively. Compared to the small database, the performance for the larger database drops much more significantly for the workloads that have a higher read-only component. Reads become a lot more expensive, because they go to disk much more often, while the cost of writes remains roughly the same. Due to the limitations of MySQL with BDB transactional tables, we could not get reproducible results for the large database with full transaction semantics.

\section{Bottleneck Analysis of Auction Site}

Figure 9 shows the number of interactions per minute for each workload as a function of the number of clients. The peak throughput for the browsing mix is 8,520 interactions per minute with 800 clients, while the bidding mix achieves a peak of 9,780 interactions per minute with 1,100 clients. Figure 9 also shows the throughput using transactions on the database server. The browsing mix shows throughput comparable to the throughput obtained without transactions up to 600 clients, and then peaks at the slightly lower value of 7,740 interactions per minute with 800 clients. Due to transaction scaling limitations of MySQL in the presence of high update rates, we are not able to present results for the case of the bidding mix with transactions.

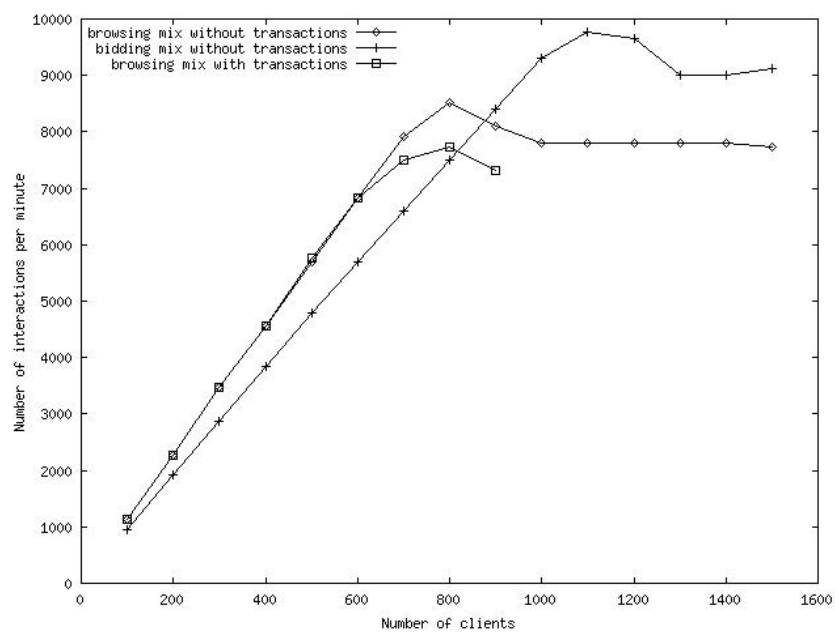

Figure 9. Auction site throughput in interactions per minute as a function of number of clients.

Figure 10 and figure 11 show the CPU utilization for the browsing and bidding mix, respectively. CPU utilization increases linearly with the number of clients on both the Web server and the database server, but it increases much more rapidly on the Web server. CPU utilization on the Web server at the peak throughput point is $100 \%$ for both workload mixes. On the database server, 
CPU utilization at the peak throughput is $58 \%$ for the bidding mix and $29 \%$ for the browsing mix.

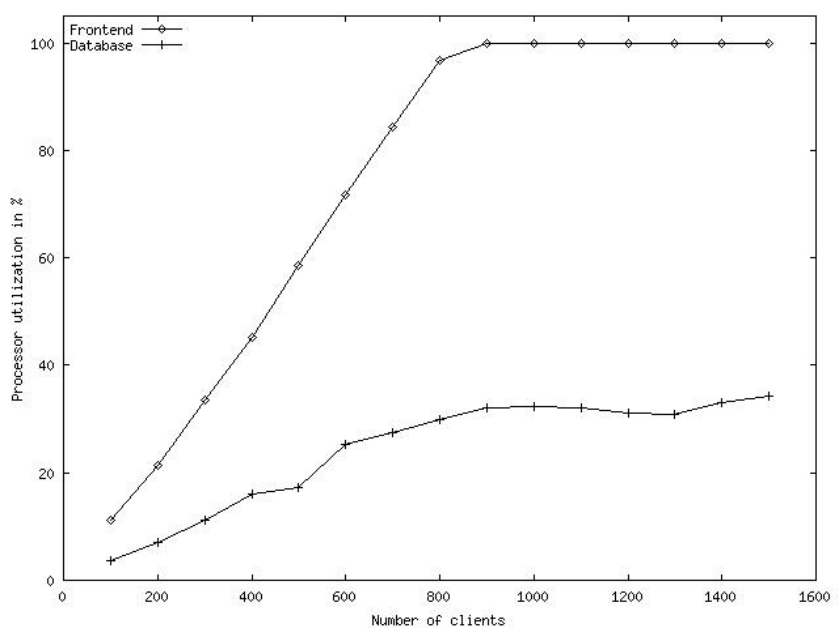

Figure 10. Auction site percentage CPU utilization as a function of number of clients for the browsing mix.

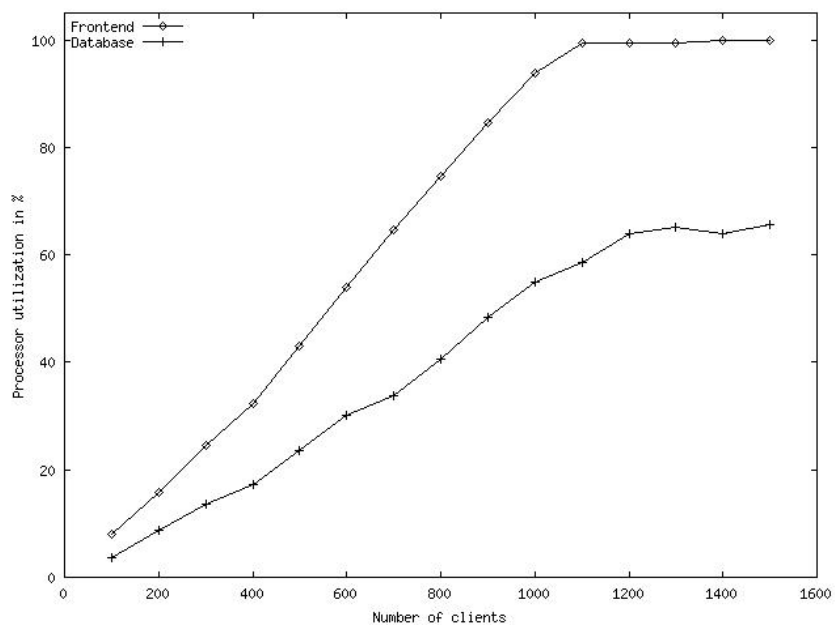

Figure 11. Auction site percentage CPU utilization as a function of number of clients for the bidding mix.

Memory and disk usage on the Web server and the database server are reported in figure 12 and figure 13, respectively. On the Web server machine, at the beginning of the experiment, a lot of Web server processes are created and the scripts are read from the disk. This explains the initial disk activity and the sharp rise in memory use. After this startup phase, there is little disk activity and memory usage remains constant at a modest 70 MB. A similar phenomenon occurs on the database server. When the first requests start arriving at the database, there is a lot of disk activity and memory use increases rapidly, until the point in time at which most of the working set (indices and frequently used tables) is in memory. After that, disk usage falls off and memory usage remains stable at around $250 \mathrm{MB}$, a relatively small value and certainly within reasonable bounds for a server machine. Although the database itself is relatively large, the temporal locality of the information in the database causes the working set to be relatively small.

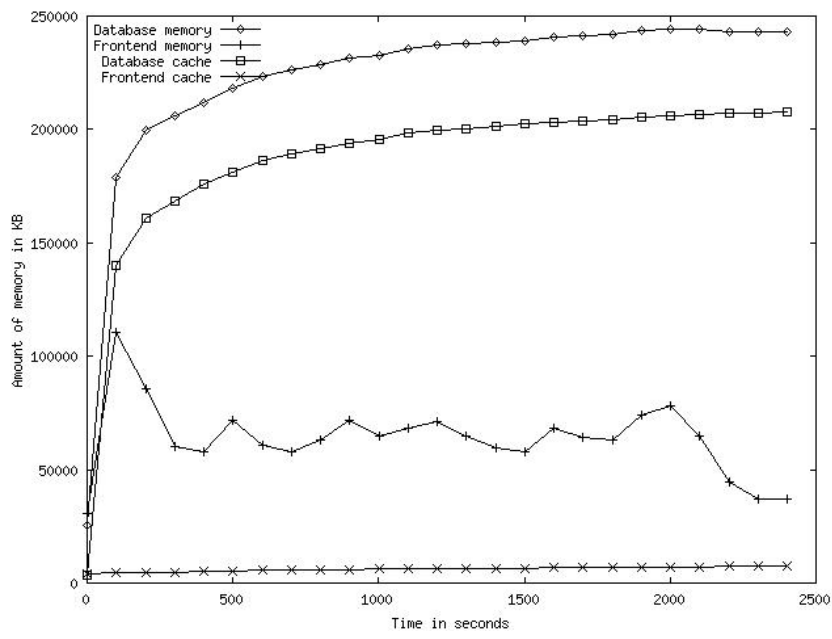

Figure 12. Auction site memory usage in $\mathrm{KB}$ as a function of time at the peak throughput for the browsing mix.

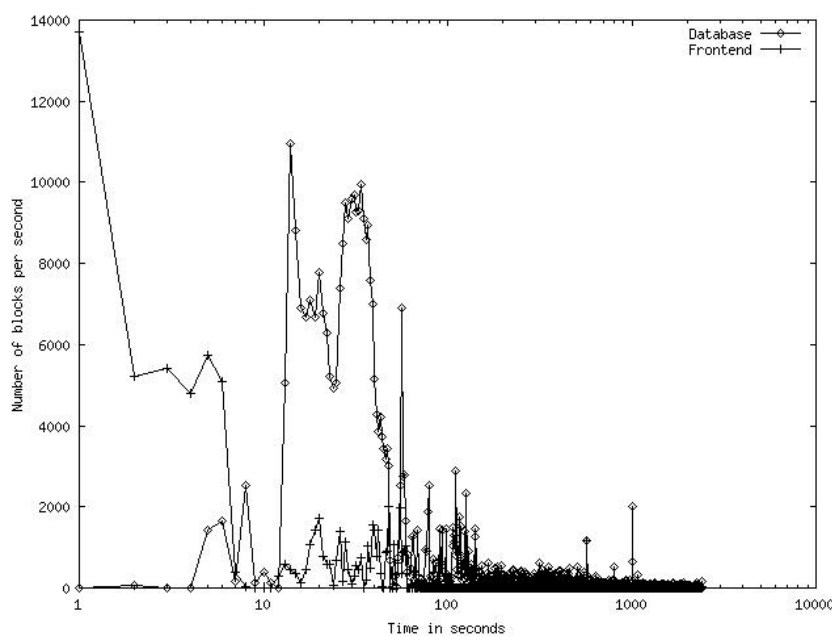

Figure 13. Auction site disk usage in number of blocks per second as a function of time ( $\mathrm{x}$-axis is log-scale) at the peak throughput for the browsing mix.

Figure 14 shows the network utilization at the peak throughput point between the clients and the Web server, and between the Web server and the database server, as a function of time. During steady state, the bandwidth between the clients and the Web server is about $55 \mathrm{Mb} / \mathrm{s}$, while the bandwidth between the Web server and the database server is about $2 \mathrm{Mb} / \mathrm{s}$. Therefore, network bandwidth is never the bottleneck for this application. 


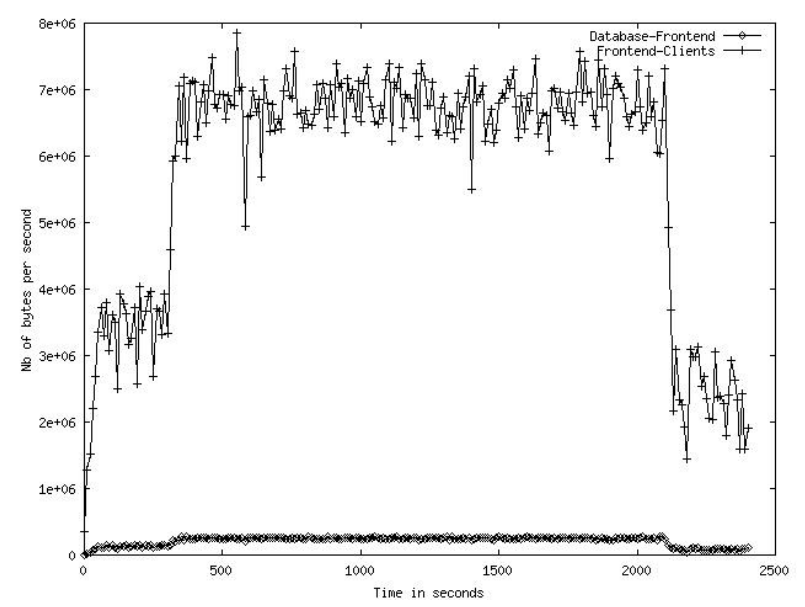

Figure 14. Auction site network usage in bytes/s as a function of time at the peak throughput for the browsing mix.

In summary, the Web server CPU is the bottleneck resource for the auction site. With transactions, however, both servers are saturated at the peak throughput for the browsing mix. We would expect that using a more complex business logic or adding features like user preferences to customize the look-and-feel of the pages sent to clients would further increase Web server CPU load. Possible solutions to this bottleneck include using an SMP or a cluster as the Web server. We have experimented with a dual-processor node for the Web server, which was sufficient to make the database CPU the bottleneck for the bidding mix.

\section{Bottleneck Analysis of Bulletin Board}

Figure 15 presents the throughput in number of interactions per minute for the browsing and submission mixes as a function of the number of clients. The browsing mix peaks at 8,160 interactions per minute with 900 clients, the submission mix at 8,580 interactions per minute with 1,000 clients. Transactions are rarely used in connection with bulletin board sites, so we do not report results with full transactional semantics for this application.

Figure 16 and figure 17 show that for both the browsing and the submission mix, the Web server CPU is the bottleneck resource at the peak throughput point. For the submission mix, the situation reverses, however, just after the peak point. The database CPU utilization jumps from $52 \%$ for 900 clients to $100 \%$ with 1,100 clients. The comment table is the main bottleneck. This large table is involved in most of the browsing and update requests.

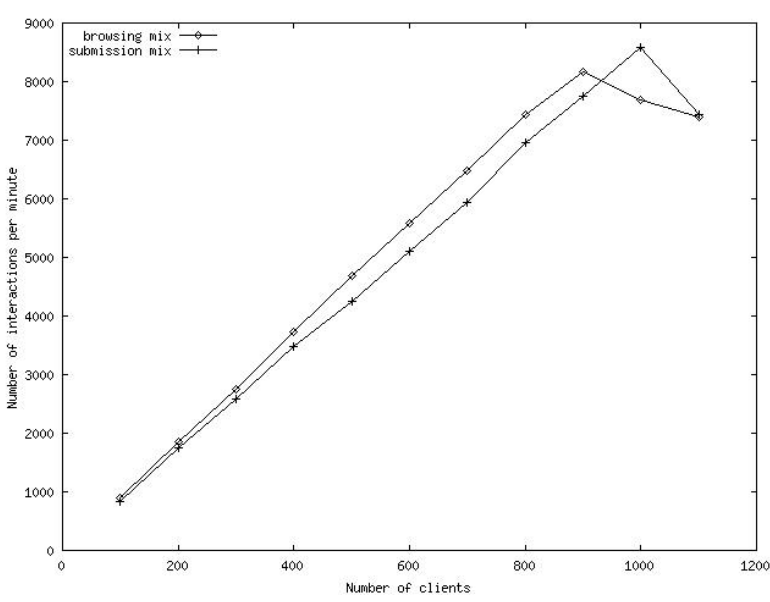

Figure 15. Bulletin board throughput in interactions per minute as a function of number of clients.

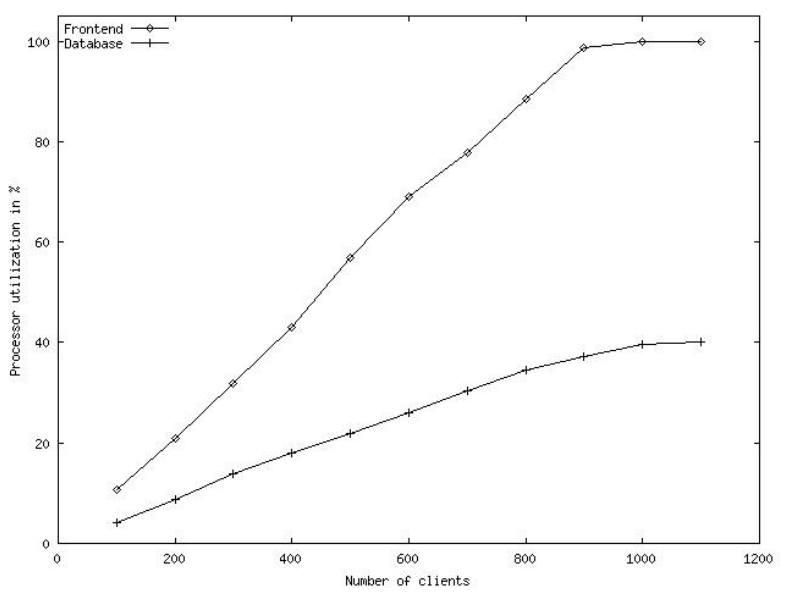

Figure 16. Bulletin board percentage CPU utilization as a function of number of clients at the peak throughput for the browsing mix.

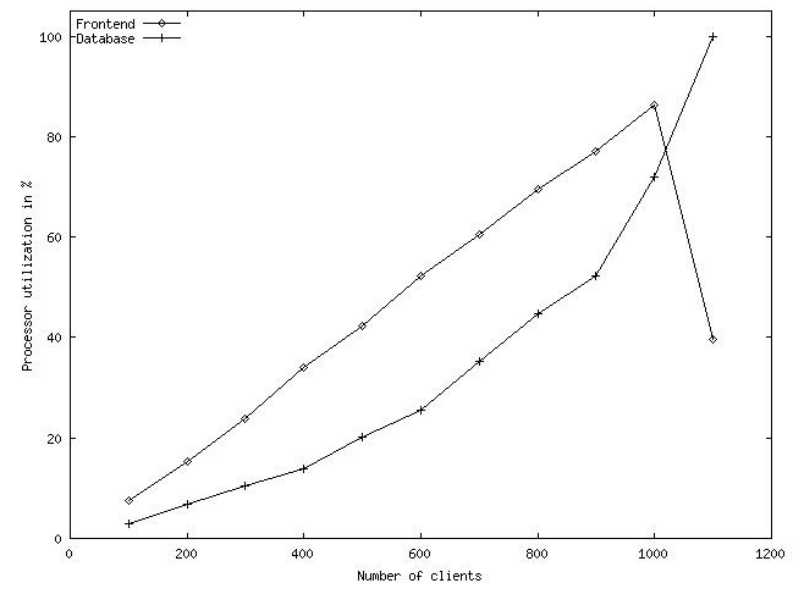

Figure 17. Bulletin board percentage CPU utilization as a function of number of clients at the peak throughput for the submission mix. 
Figure 18 and figure 19 report on memory and disk usage. As with the auction site, we observe a burst of reads at startup. With the auction site, clients only access new items, and therefore the working set is limited to the new items and fits in memory. With the bulletin board site, however, clients also continue to access old stories and comments. The disk reads after the initial startup are largely due to accesses to old stories. The memory utilization increases correspondingly. Due to the larger amount of data manipulated, the database server requires an average of $350 \mathrm{MB}$, while Web server memory usage remains modest at $70 \mathrm{MB}$.

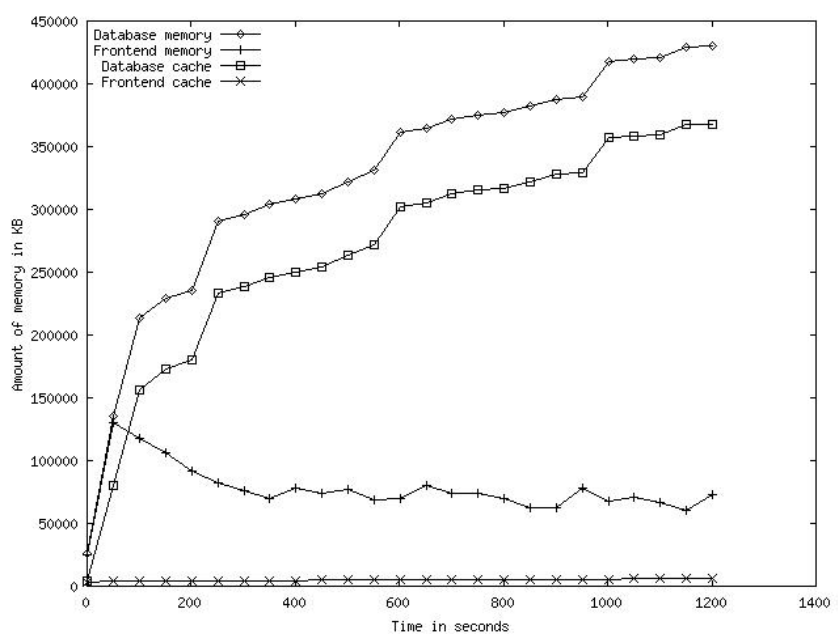

Figure 18. Bulletin board memory usage in KB as a function of time at the peak throughput for the browsing mix.

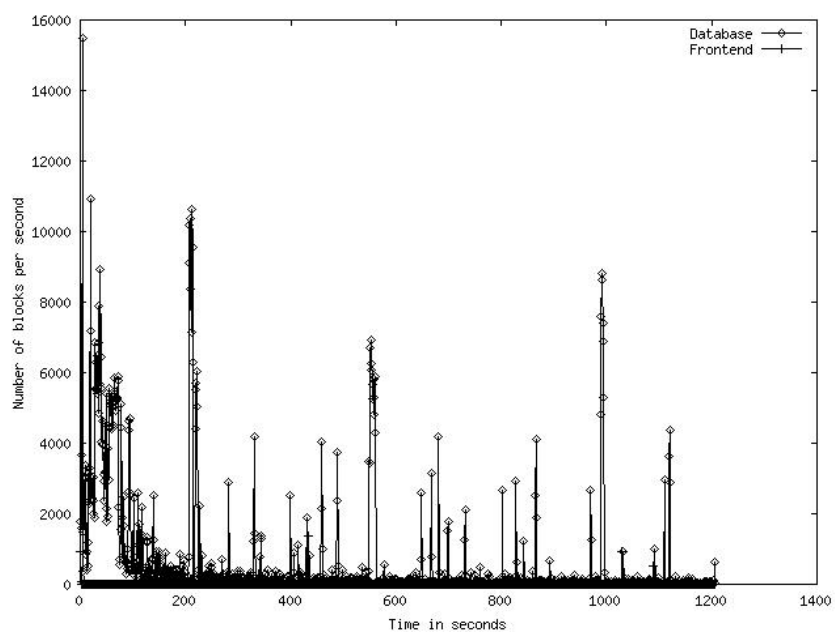

Figure 19. Bulletin board disk usage in number of blocks per second as a function of time at the peak throughput for the browsing mix.

Figure 20 shows that the network traffic between the database and the Web server is much higher than for the other sites $(9 \mathrm{Mb} / \mathrm{s}$ on average). The network traffic between the clients and the Web server is $20 \mathrm{Mb} / \mathrm{s}$. In any case, the network bandwidth is not a bottleneck.

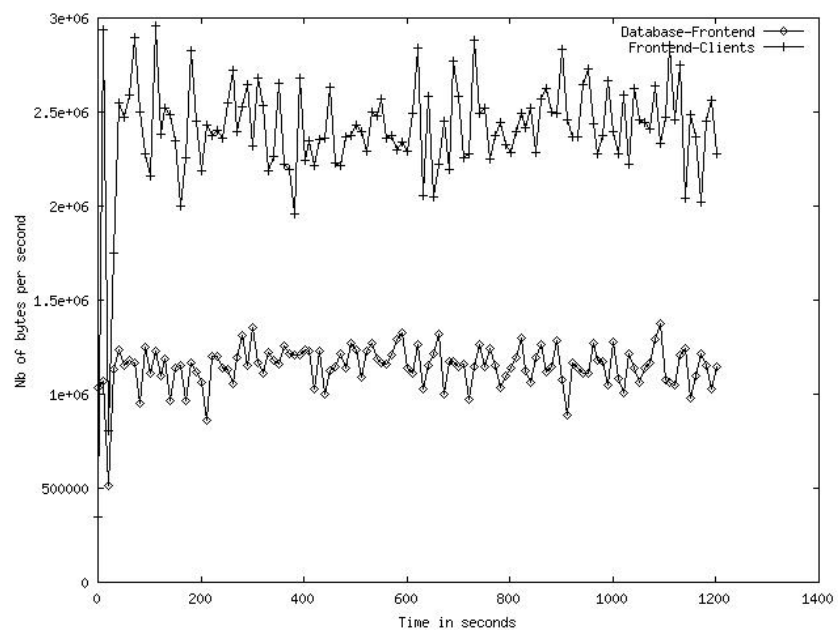

Figure 20. Bulletin board network usage in bytes/s as a function of time at the peak throughput for the browsing mix.

To summarize, the Web server CPU is the bottleneck resource at the peak point for the bulletin board, for both workload mixes. The database CPU approaches saturation as well at peak throughput and becomes the bottleneck if even more clients are added. A possible approach to offloading the database is to generate static pages for the stories of the day or the most recent stories. We experimented with this technique, and found that it made the Web server the bottleneck under all circumstances.

\section{Related Work}

For static Web content, the presence of a number of agreed upon benchmarks, such as, e.g., the NLANR traces [17] and the Polygraph benchmark [26], have greatly fostered research in systems support for static Web content servers, including OS support, caching, and clustering. Similar studies for dynamic content Web sites have been far fewer, and their results much more difficult to compare, in our opinion, in part because of the lack of benchmarks. Specweb99 [28] is a first-generation dynamic content benchmark. The set of benchmarks introduced in this paper is more representative of the diversity of current dynamic content. Zhang et al. [27] study load balancing among machines in a dynamic content Web server cluster, but their study uses a readonly workload, avoiding any issues of consistency maintenance in the presence of writes. Ji et al. [11] use simulation of a white pages server and an auction site to study caching of database results. Our benchmarks allow measurement of system overheads on real systems rather than simulation. The Neptune project [19] studies 
scalability of clusters for dynamic content, but does not include benchmark specifications, or a bottleneck analysis like the one presented in this paper.

Menascé et al. model client workloads for dynamic content sites [13]. Starting from access logs of an actual ebusiness site (an auction site that sells domain names), they have developed detailed models of customer behavior, and resource management methods to optimize site revenue [14]. For the online bookstore, we adopt the workload from the one specified by TPC-W. For the other applications, we adopt similar workload models. One possible avenue of further work is to investigate the effect of these more sophisticated workload models on the performance of the applications.

Cain et al. [4] present a detailed architectural evaluation of TPC-W implemented using Java servlets. They investigate the impact of Java servlets on the memory system, the branch predictor, and the effectiveness of coarse-grain multithreading. Our study is focused instead on understanding bottlenecks at the node level.

\section{Conclusions}

We have presented three benchmarks for dynamic content sites with very different characteristics: an online bookstore, an auction site, and a bulletin board. For the online bookstore, we follow the specification provided by TPC-W. For the auction site and the bulletin board, we provide our own specifications.

We have implemented the three dynamic content benchmarks and a workload generator tool that allows us to vary the workload driving the dynamic content server. We have used our implementations to carry out a bottleneck characterization of the benchmarks. Different benchmarks show different bottlenecks: the database CPU for the online bookstore, and the Web server CPU for the auction site and the bulletin board. Complex queries cause the database CPU to be the bottleneck for the online bookstore. In contrast, the queries for the other applications are simpler.

We are making the source code of our implementations freely available on our Web site. We hope other researchers will use them, making performance results of dynamic content Web sites more reproducible and easier to compare.

\section{References}

[1] The Apache HTTP Server Project - http://httpd.apache.org/. [2] Apache Tomcat - http://jakarta.apache.org/tomcat/.

[3] L. Breslau, P. Cao, L. Fan, G. Phillips and S. Shenker - Web Caching and Zipf-like Distributions: Evidence and Implications - Proceedings of the IEEE Infocom Conference, 1999.
[4] Harold W. Cain, Ravi Rajwar, Morris Marden and Mikko H. Lipasti - An Architectural Evaluation of Java TPC-W Proceedings of the Seventh International Symposium on HighPerformance Computer Architecture, 2001.

[5] Emmanuel Cecchet, Anupam Chanda, Sameh Elnikety, Julie Marguerite and Willy Zwaenepoel - A Comparison of Software Architectures for E-business Applications - Rice University Technical Report TR02-389, 2002.

[6] Emmanuel Cecchet, Julie Marguerite and Willy Zwaenepoel - Performance and scalability of EJB applications - $17^{\text {th }}$ Annual Conference on Object-Oriented Programming, Systems, Languages, and Applications, OOPSLA 2002.

[7] eBay - http://www.ebay.com/.

[8] Enterprise JavaBeans Technology http://java.sun.com/products/ejb/.

[9] Java Servlet Technology http://java.sun.com/products/servlet/index.html/.

[10] JBoss - http://www.jboss.org/.

[11] Minwen Ji, Edward W. Felten, Jaswinder Pal Singh and Mao Chen - Query Affinity in Internet Applications - Computer Science Technical Report, Princeton University, 2001

[12] JOnAS - http://www.objectweb.org/jonas/.

[13] Daniel Menascé, Flavia Ribeiro, Virgilio Almeida, Rodrigo Fonseca, Rudolf Riedi and Wagner Meira Jr - In Search of Invariants for E-Business Workloads - Proceedings of EC'00, 2000.

[14] Daniel Menascé, Rodrigo Fonseca, Virgilio Almeida and Marco Mendess - Resource Management Policies for Ecommerce Servers - Second Workshop on Internet Server Performance WISP'99, 1999.

[15] MySQL - http://www.mysql.com/.

[16] Netcraft Web Server Survey, September 2002 http://www.netcraft.com/survey/.

[17] NLANR - http://pma.nlanr.net/Traces/.

[18] PHP Hypertext Preprocessor - http://www.php.net/.

[19] Kai Shen, Tao Yang, Lingkun Chu, JoAnne L. Holliday, Doug Kuschner, Huican Zhu - Neptune: Scalable Replica Management and Programming Support for Cluster-based Network Services $-3^{\text {rd }}$ USENIX Symposium on Internet Technologies and Systems (USITS), 2001.

[20] Slashcode - http://www.slashcode.org/.

[21] Slashdot - http://www.slashdot.org/.

[22] Handling the Loads - Slashdot http://slashdot.org/article.pl?sid=01/09/13/154222\&mode $=$ thread\&tid=124.

[23] Sysstat package - http://freshmeat.net/projects/sysstat/. [24] Transaction Processing Performance Council http://www.tpc.org/.

[25] TV Show Message Board http://mcbeal.hypermart.net/stats/

[26] Web Polygraph - http://www.web-polygraph.org.

[27] Xiaolan Zhang, Michael Barrientos, J. Bradley Chen and Margo Seltzer - HACC: An Architecture for Cluster-Based Web Servers - Proceedings of the 2000 Annual Usenix Technical Conference, 2000.

[28] SPECweb99 Benchmark - Proceedings of the $2^{\text {nd }}$ Workshop on Workload Characterization, 1999. 